\title{
Removing Atmospheric Turbulence Using Restoration Algorithm
}

\author{
${ }^{1}$ Vani.M, ${ }^{2}$ Shruthi.M.K \\ ${ }^{1} 4^{\text {th }}$ Sem M.Tech Dept Of CS\&E, SJMIT, Chitradurga ${ }^{2}$ Asst. Professor, Dept of CS\&E, SJMIT, Chitradurga, \\ Visvesvaraya Technological University "Jnana Sangama”, Belgaum, India
}

\begin{abstract}
It remains a challenge to simultaneously remove geometric distortion and space-time-varying blur in frames captured through a turbulent atmospheric medium. To solve, or to reduce these effects, we proposed a new approach in this paper for restoring a single high-quality image from a given image sequence distorted by atmospheric turbulence. This approach is used to reduce the space and time-varying deblurring problem to a shift invariant one. First we use a B-spline based non-rigid image registration algorithm to register each observed frame with respect to a reference image and it also suppresses geometric deformation. Next approach is image stitching algorithm in that the frames are stitched together using an edge-correlation algorithm. Finally, a sharpening high-pass filter is applied to the final image to generate a final high-quality output. This approach can effectively alleviate blur and distortions, recover details of the scene, and significantly improve visual quality.
\end{abstract}

\section{Introduction}

Atmospheric turbulence is one of the sources of distortion that can degrade the quality of images and videos acquired by cameras viewing scenes from long distances. This phenomenon is especially common in astronomy. For examples, stars in outer space viewed through telescopes appear blurred since the earth's atmosphere de-grades the image quality. The physical cause of the turbulence is the fluctuations in the refractive index of air. These fluctuations involve many factors including wind velocity, temperature gradients, elevations, etc. The main factor is temperature variation, but it is difficult to measure these factors, and the parameters which are associated with a true physical model of atmospheric turbulence are typically unknown. Consequently the task of compensating for turbulence distortion can be viewed as a blind restoration problem. In addition to blurring, another effect associated with turbulence is geometric distortion, which arises because the turbulence is time and space varying.

The performance of long-distance imaging system can be affect due to the atmospheric turbulence caused by variation of refractive index along the optical transmission path [1][2][3]. It produces geometric distortion, motion blur, and sometimes out-of-focus blur when the turbulence is violent.

The imaging process can be modelled as:

Where

$$
\mathrm{gk}=\mathrm{BkFk} Z+\varepsilon(1)
$$

$\mathrm{Z}=$ Denotes the ideal image without turbulence distortion or blur.

$\mathrm{Fk}=$ Represent the deformation matrix.

$\mathrm{Bk}=$ Represent the blurring matrix.

$\varepsilon=$ Denotes additive noise.

$\mathrm{gk}=$ Represent the $\mathrm{k}$-th observed frame.

Several approaches have been proposed to solve this problem [4][8][9] and since the information in a single frame is usually insufficient to recover, all these works are based on videos or image sequences under the assumption that the scene is static. For example [7] provides an experiment to illustrate that the local turbulent motion has a zero-mean Gaussian distribution, and we need to averaging the observed frames in order to remove geometric distortion. Such averaged image, though even more blurred then the original data, can serve as a reference frame to register each observed frame

Current restoration algorithms for atmospheric imaging systems can generally be divided into two main categories. One important category is based on multi-frame reconstruction. This type of approach first requires a non-rigid image registration technique to register each observed frame and further estimate the deformation matrix Fk by using the registration parameters. Then a sharp image is reconstructed under a Bayesian image reconstruction framework. In [7] Shimizu and Okutomi proposed an improved B-spline based non-rigid registration method for the turbulence distortion estimation. They introduced a stabilization term into the cost function, and claimed that this term enables more accurate estimation in the regions containing less texture. A Gauss-Newton method was employed to optimize their cost function. However, direct implementation of the 
algorithmic procedure of the registration method described in5 is time consuming. Besides, the Bayesian reconstruction method proposed in 5 is also sensitive to the assumed model of noise and the registration error.

Another class of approaches employs image selection and fusion methods to reduce the blurring effects caused by turbulence [5]. The frame selection technique finds out frames of the best quality (lucky frame) from a short exposure video stream, and the output image is produced by fusing these lucky frames [8]. The problem of this approach is the very low probability of existence of a whole high-quality frame. To alleviate this problem Vorontsov introduced the idea of "lucky region"[9][10] where it is shown that for short-exposure images turbulence creates "mutations" in local image quality, and randomly makes some regions sharper(see examples in Fig.1), which are called lucky regions. The lucky regions can be detected by a sharpness metric, and then fused to produce a high quality image [9][10]. A difficulty with this method is again the low probability of the appearance of lucky regions. Although high-quality frames, this method generally requires a large number (usually hundreds) of frames to create one single sharp image. Another problem is that this method is applicable only for short-exposure videos, while for long-exposure videos motion blur makes the lucky regions too rare to be useful.

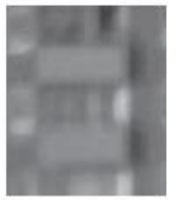

(a) frame 1

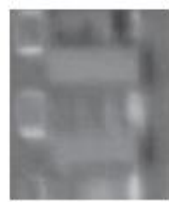

(b) frame 4

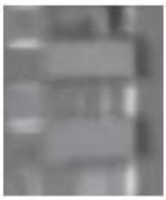

(c) frame 11

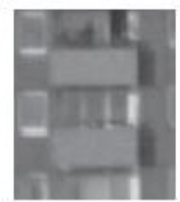

(d) frame 23

Fig.1. Isoplanatic patches from the short exposure image sequence building taken through hot air, where a variation of blur can be observed. (b) Illustrates a lucky frame example which is much sharper than the others.

Due to the limited Field-of-View (FOV) of a single camera, it is sometimes desired to extend the FOV using multiple cameras. Image stitching is one of the methods that can be used to exploit and remove the redundancy created by the overlapping FOV. However, the memory requirement and the amount of computation for conventional implementation of image stitching are very high. In this project, this problem is resolved by performing the image stitching and compression in a strip-by-strip manner. First, the stitching parameters are determined by transmitting two reference images to an intermediate node to perform the processing. Then, these parameters are transmitted back to the visual node and stored in there.

These parameters will be used to determine the way of stitching the incoming images in a strip-by-strip manner. After the stitching of a strip is done, it can be further compressed using a strip-based compression technique. Most of the existing methods of image stitching either produce a 'rough' stitch that cannot deal with common features such as blood vessels, comet cells and histology, or they require some user input. Approaches for image stitching that optimize the search for the best correlation point by using Levenberg-Marquardt method gives good results, but it is computationally expensive and can get stuck at local minima. The approach offered in this project makes the selection of the best correlation point in the following way. Based on knowledge about the expected overlap when using the motorized stage, it would be straightforward to find the best correlation point in the ideal casse. However the overlap area is not perfect and not accuracy for one pixel, due to deviations in stage position from the ideal and due to stage/camera misalignment.

Our algorithm offers a way to overcome this problem by searching the small area around the expected central overlap pixel in order to find the best correlation point. Positioning of acquired images with a manual stage is much less accurate, so there is a need to search a wider area in order to find the best cross-correlation point. This project's goal is to create a Mat-lab script that will stitch two images together to create one larger image. Image stitching has wide uses in photo applications and has become a required toolset for many photographers. These stitched images, become panoramic views which increase the visual aesthetics of a scene, and are widely sought out for posters, postcards, and other printed materials. This project will be performed using point correspondences between the two images and utilizing Matlab's Image Processing Toolbox.

\section{Proposed System}

To correct geometric distortion and reduce space and time varying blur, we are proposing a new approach in this work capable of restoring a single high-quality image from a given image sequence distorted by atmospheric turbulence. This approach uses two major steps. For the first step, we use a B-spline based nonrigid image registration algorithm to register each observed frame with respect to a reference image. To improve registration accuracy, a symmetry constraint is introduced to the cost function to penalize inconsistency between the forward and backward deformation parameters during the estimation process. A fast Gauss-Newton method is used to reduce the computational cost of the registration algorithm. 
Our new idea and an addition to this approach involve separating a single frame into a set of overlapping sub-frames and running the earlier-described algorithm on those sub-frames. In addition to reducing computational load and time, this improves on the original method by reducing the effect of symmetric errors on the frame, since the errors are localized and isolated to the particular block rather than affecting the whole image. Another benefit of this points approach is that scaling the number of the test points causes a much smaller processing time increase as opposed to the processing time that would be required for the same number of test points if applied to the whole image. The second step of our proposal is to stitch the undeformed back into a single image and then, using a high-pass sharpening filter produces a final, high-quality image.

\section{Proposed Approach}

The proposed approach contains restoration algorithm which includes three main steps see the diagram in Fig. 2).

A. Non-rigid image registration.

B. Image stitching algorithm.

C. A sharpening high-pass image filtering method.

Step A: Given an observed sequence $\{\mathrm{gk}\}$, step1 of our proposed approach registers each frame onto a fixed reference grid, generating a new sequence $\{\mathrm{Rk}\}$ without geometric deformation. This process makes sure that for a given pixel located at a position, say $\mathrm{x}$ the most correlated pixels in each frame across time are aligned at this same position. This step improves the performance of the subsequent temporal filtering.

Step B: Restores a single image from the registered sequence $\{\mathrm{Rk}\}$ by using image stitching algorithm.

Step C: A sharpening high-pass filter method produces a high quality image.

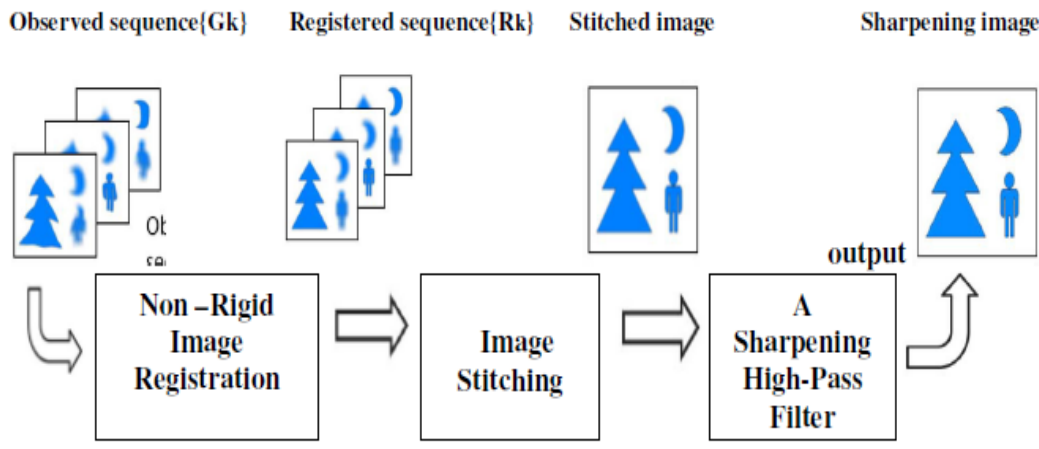

Fig. 2 Block diagram for the proposed framework

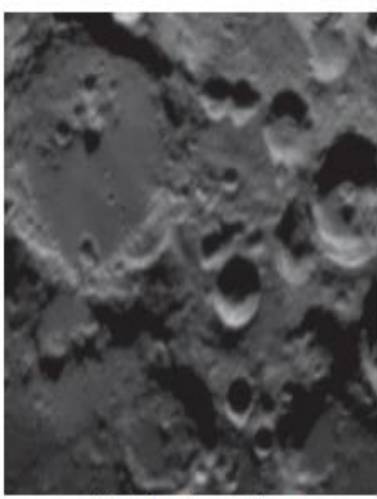

(a) One observed frame

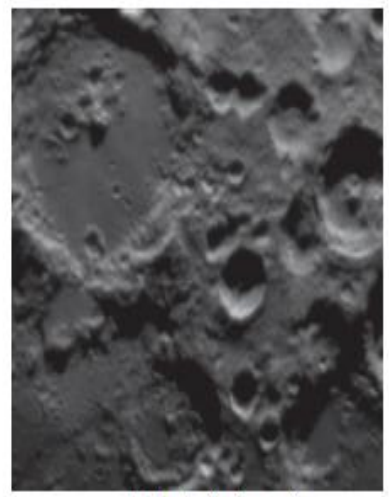

(b) Lucky fusion region

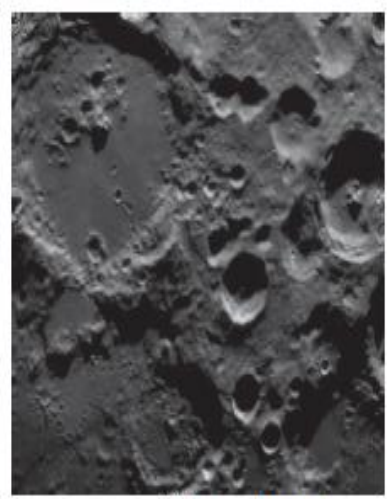

(c) Proposed approach

Fig 3. Image reconstruction results using 20 frames taken from the video Moon Surface distorted by real atmospheric turbulence. 


\section{A. Non-rigid Image Registration}

Assume ' $\mathrm{g}$ ' represents an observed distorted image, and ' $\mathrm{R}$ ' denotes a reference image without turbulent deformation (which can be obtained by averaging the frame sequence). A non-rigid deformation model can be employed to describe the geometric distortions between these two images. In this model a complex motion is represented by the movements of ' $n$ ' control points whose initial position $\widetilde{X}_{\sigma i}=\left(\widehat{x}_{o i} \widehat{y}_{\sigma i}\right)^{T}(\mathrm{i}=1 \ldots \mathrm{n})$ are equally spaced on the given image ' $\mathrm{g}$ '.

The displacement of all the control points on the reference image ' $R$ ' is denoted as the deformation vector:

$$
\vec{P}=\left[\Delta \hat{x}_{1, \ldots \ldots, n} \Delta \hat{x}_{n} \Delta \hat{y}_{1}, \ldots, \Delta \hat{y}_{n}\right]^{T}
$$

Where $\Delta \widehat{\boldsymbol{x}}$ and $\Delta \widehat{\boldsymbol{y}}$ are the horizontal and vertical displacement from the initial position. The deformed position of any given pixel located at $\mathrm{X}=(x, y)^{T}$ from image $\mathrm{G}$ can then be described as:

$$
\mathrm{W}(\mathrm{X} ; \vec{P})=\mathrm{X}+\mathrm{A}(\mathrm{X}) \vec{P}
$$

Where $\mathrm{A}(\mathrm{X})$ denotes the basis function matrix for $\mathrm{X}$ :

$$
\mathrm{A}(\mathrm{X})=\left[\begin{array}{cc}
c 1 \ldots \ldots c n & 0 \ldots \ldots \\
0 \ldots \ldots & c 1 \ldots \ldots c n
\end{array}\right]
$$

and $\mathrm{A}(\mathrm{X}) \vec{P}$ is the motion vector, which is a linear combination of the movements of all control points. The weight or spline basis.

$c_{\tilde{i}}$ is determined by the distance between $\mathrm{X}$ and $\widetilde{X}_{\text {or }}$ using B-spline interpolation:

$$
\begin{gathered}
c_{\mathrm{i}}=\beta\left(\frac{x-\hat{x}_{0 \mathrm{fi}}}{h_{w}}\right) \beta\left(\frac{y-\hat{p}_{\mathrm{oi}}}{h_{y}}\right) \\
\beta(\mathrm{t})=\left\{\begin{array}{cc}
2 / 3-(1-|\mathrm{t}| / 2) \mathrm{t} 2, \text { if } 0 \leq\|\mathrm{t}\| \leq 1 \\
(2-|\mathrm{t}|) \mathrm{y} / 6, & \text { if } 1<\|\mathrm{t}\|<2 \\
0, & \text { otherwise }
\end{array}\right.
\end{gathered}
$$

Where $h_{z}$ and $h_{y}$ are the horizontal and vertical intervals between neighboring control points.

In the classic B-spline based registration approach, the deformation vector can be estimated by minimizing the following cost function:

$$
\mathrm{C}(\overrightarrow{\mathrm{P}})=\sum_{\mathrm{X}} \mid R\left(W(\mathrm{X}, \vec{P})-\left.\mathrm{G}(\mathrm{X})\right|^{2}\right.
$$

However, because the above function lacks prior constraint, the resulting estimate is unstable and gets easily stuck in local minima. To improve this method, a stabilization constraint is proposed in [7], which makes the estimated deformation parameters remain small in the regions that have less texture (low image gradient). However, we know that the deformation caused by atmospheric turbulence is independent from image content. In other words, such stabilization constraint would bring unnecessary bias into the estimation.

To accurately estimate the deformation vector and further the motion vectors from image $\mathrm{R}$ to $\mathrm{G}$, we instead introduce a symmetry constraint into the B-spline registration algorithm. This constraint is based on an important property that the registration should be symmetric or inverse consistent. Let $\vec{P}$ denote the Deformation vector that can transform R into G (forward deformation), and $\bar{P}$ denote the inverse vector that transform G into $\mathrm{R}$ (backward deformation). Then approximately we have: $\vec{P}=-\vec{P}$. Combing the two sets of vectors into one vector:

$$
P^{T}=\left[\bar{P}^{T}, \overrightarrow{\mathrm{P}}^{T}\right]
$$


The proposed cost function to minimize becomes:

$\mathrm{C}(\overrightarrow{\mathrm{P}})=\sum_{x} \mid R\left(W(\mathrm{X} ; \vec{P})-\left.\mathrm{G}(\mathrm{X})\right|^{2}+\sum_{x} \mid G\left(W(\mathrm{X} ; \vec{P})-\left.\mathrm{R}(\mathrm{X})\right|^{2}+\gamma(\overrightarrow{\mathrm{P}}+\bar{P})^{T}(\overrightarrow{\mathrm{P}}+\bar{P})\right.\right.$

Where $\gamma$ scalar controls the effect of symmetry constraint.

Gauss-Newton method is used to optimize the above $\mathrm{C}(\mathrm{p})$, and the update of the parameter set $\mathrm{P}$ can be derived as follows:

$$
\begin{aligned}
& P^{l+1}=P^{I}-H^{-1} \mathrm{~b}
\end{aligned}
$$

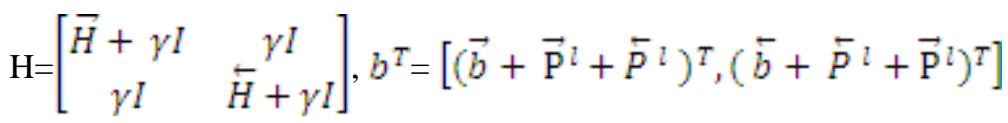

$$
\begin{aligned}
& \vec{H}=\sum_{X} \bar{d}(X) \bar{d}(X)^{T}, \bar{H}=\sum_{X} \bar{d}(X) \bar{d}(X)^{T} \\
& \vec{b}=\sum_{R} \vec{d}(\mathrm{X})\left[R\left(W\left(X_{;} \vec{P}^{\mathrm{I}}\right)\right)-\mathrm{G}(\mathrm{X})\right], \Sigma_{\mathbb{R}} \vec{d}(\mathrm{X})\left[G\left(W\left(X_{;}, \bar{P}^{\mathrm{l}}\right)\right)-\mathrm{R}(\mathrm{X})\right]
\end{aligned}
$$

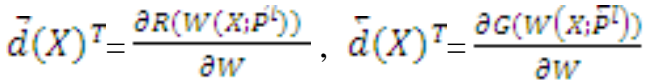

In the above process, the computational cost for calculating the matrices $\vec{H}$ and $\bar{H}$ is tremendous if it is implemented directly. For instance, assuming that the size of both R and G is $320 \times 240$ and the intervals $h_{x}=$ $h_{y}=16$, we have around 300 control points, and the size of $\overrightarrow{\mathrm{P}}$ would be $600 \times 1$. In Eq(10) the matrix product $\vec{d}(\mathrm{X}) \vec{d}(X)^{T}$ would cost at least 360,000 multiplication operations, and this process needs to be repeated for all the $320 \times 240$ pixels in the image. Furthermore, if the size of the image increases with the intervals fixed, the corresponding number of the control points will increase, which means the computational complexity in calculating $\vec{d}(\mathrm{X}) \vec{d}(X)^{T}$ for each pixel will also be raised.

To solve this problem, we use a fast implementation method for calculating $\vec{H}$ and $\bar{H}$. Take $\vec{H}$ for instance. The gradient of image $\mathrm{R}$ at position $\mathrm{W}(\mathrm{X} ; \vec{P})$ can be denoted as:

$$
\frac{\partial R\left(W\left(X_{w} \overline{\mathrm{P}}\right)\right)}{\partial W}=\left\lfloor r_{x}(W), r_{y}(W)\right\rfloor
$$

We define B-spline basis vector $c_{X}=\left[c_{1}, \ldots \ldots, c_{n}\right]^{T}$, and matrix $C_{X}=c_{X} c_{X}{ }^{T}$. Then $\vec{H}$ can be computed as shown below:

$$
\vec{H}=\sum_{X} \overrightarrow{H_{Z}}
$$

Where

$$
\overrightarrow{H_{X}}=\left[\begin{array}{cc}
r_{z}(W)^{2} C_{X} & r_{z}(W) r_{y}(W) C_{X} \\
r_{z}(W) r_{y}(W) C_{X} & r_{y}(W)^{2} C_{X}
\end{array}\right]
$$

In the $\mathrm{Eq}(5)$, it can be observed that $\beta(t)=0$ if $|t| \geq 2$, which means the value of each pixel can only influence the neighboring $4 \times 4$ control points in the update procedure. So, for the pixel at position $\mathrm{x}$ the basis vector $C_{Z}$ contains at most 16 non-zero entries. In other words, $C_{Z}$ is a sparse matrix with 256 non-zero entries, and among them there are only 136 unique values due to the symmetry of $C_{R}$.

Hence we can see that each $\vec{H}$ contains 1024 non-zero entries, and requires 680 multiplications (136 for calculating $C_{X}$, and 544 for calculating $\overrightarrow{H_{M}}$ through Eq. (15)). This number is independent from either the 
number of control points or the size of the images. Implementation based on this derivation can drastically reduce the computational cost for the non-rigid registration procedure. For example, on Intel Core 2 CPU (3.00 $\mathrm{GHz}$ ) the execution time for registering $320 \times 240$ images using MATLAB is around 2 hours for direct implementation of the iteration process [9]-[11], but it only requires 10 minutes for the above new method.

\section{B. Image Stitching Algorithm}

The 3 important stages in the process of stitching images are (see Fig.4):

Image acquisition: The images that need to be stitched are acquired using a camera mounted on a tripod stand. The camera angle is varied to take different overlapping sample images.

Image registration: The process of image registration aims to find the translations to align two or more overlapping images such that the projection from the view point through any position in the aligned images into the $3 \mathrm{D}$ world is unique. Image registration consists of four main components:

- $\quad$ Feature set- The set of features includes the intensity values, contours, textures and so on. A feature set must be selected for each image registration method.

- Similarity measure- It is a function which returns a scalar value that provides an indication of the similarities between two features.

- $\quad$ Search set- It is a set of possible transformations for aligning

- Search strategy- It is the algorithm that decides how to select the next transformations from the search

set.

Image Merging: Image merging is the process of adjusting the values of pixels in two registered images, such that when the images are joined, the transition from one image to the next is invisible. It should also ensure that the new image has a quality comparable to that of the original images used. Image merging can be carried out by making the seam invisible in the output image. The seam is the line that is visible at the point where the two images overlap.

Techniques used:

Linear distribution of intensity differences.

Linear distribution of median intensity differences.

Intensity adjustment with respect to corresponding pixels in overlapping region.

\section{Sharpening High-Pass Filter Method}

An image can be filtered to remove a band of spatial frequencies such as high-frequencies and lowfrequencies. High-frequencies will increase the brightness transition of an image where as low-frequencies reduces the brightness transition of an image. The highest frequencies normally will be found at the sharp edges or points.

Spatial filtering operations include high pass, low pass and edge detection filters. High pass filters accentuate the high frequency details of image and attenuate the low frequency, creating a sharpening effect.

\section{Advantages of Proposed System}

1. The proposed algorithm uses a multi-frame reconstruction approach with the addition of sub-frame evaluations with overlap and sub-image stitching to create a final image.

2. The algorithm registers the sub-frames to suppress spatial and geometric deformation using a B-spline based non-rigid image registration method.

3. Reducing the computational load and time by removing the errors on the frame.

4. A non-rigid image registration algorithm also reduces the cost function.

5. A high-pass sharpening filter produces a final high-quality image.

6. Image stitching algorithm generates one panoramic image from a series of smaller, overlapping images.

\section{Result}

Finally we give some real data reconstruction results using the proposed method and lucky region fusion method as comparison 8-10. The first set of data shows the moon surface $(400 \times 400)$ where the scene is relatively clean, slightly blurred and with mild turbulence (see Fig. 3(a)). Only 20 frames are taken for the reconstruction experiment, so that the amount of sharp regions (lucky region) is very limited. We can see that basically lucky region fusion method failed to recover high frequency content (Fig.3(b)) while the proposed method gives significant improvement in image quality (Fig.3(c)). 


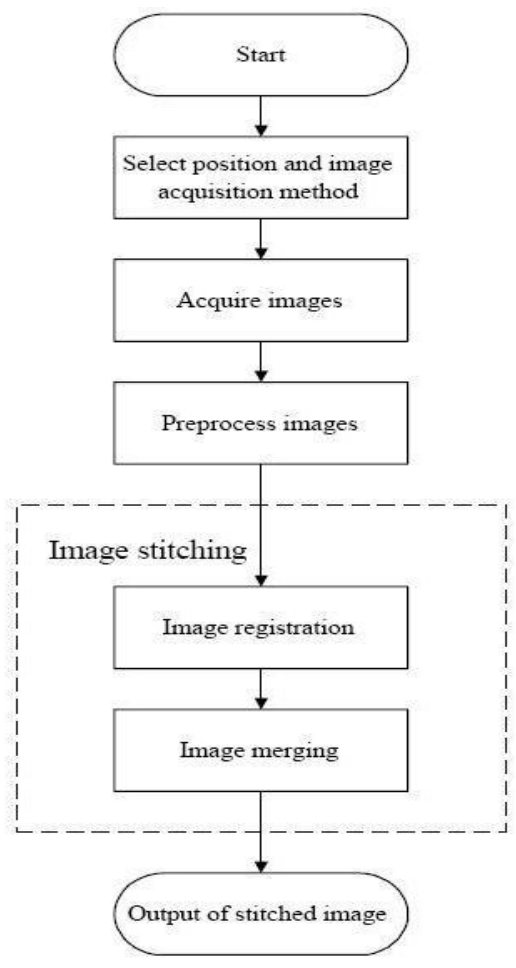

Fig. 4 Steps for image stitching

\section{Conclusion}

In this paper we proposed a new approach for restoring a single high-quality image from an image sequence distorted by air turbulence. The proposed algorithm first registers the frames to suppress geometric deformation using B-spline based non rigid image registration which includes a symmetry constraint to effectively improve the estimation accuracy. Next the frames are stitched together using an edge-correlation method. Finally a sharpening high-pass filter is applied to the final image to generate a final high-quality output. Experiments using controlled and real data illustrate that this approach is capable of alleviating geometric deformation and space-time varying blur caused by turbulence, recovering unprecedented details of the scene and significantly improving visual quality.

\section{References}

[1] W.E.K. Middleton, Vision through the Atmosphere. Univ. of Toronto Press 1958

[2] M.C. Roggemann and B.M. Welsh, Imaging through Turbulence. CRC Press, 1996.

[3] D.Li, R.M. Mersereau, and S. Simske, "Atmospheric Turbulence- Degraded Image Restoration Using Principal Components Analysis," IEEE Geoscience and Remote Sensing Letters, vol. 4, no. 3, pp. 340-344, July 2007.

[4] R.N. Tubbs, "Lucky Exposures: Diffraction Limited Astronomical Imaging through the Atmosphere," PhD thesis, Cambridge Univ, Sept. 2003.

[5] M.A. Vorontsov and G.W. Carhart, "Anisoplanatic Imaging through Turbulent Media: Image Recovery by Local Information Fusion from a Set of Short-Exposure Images,” J. Optical Soc. Am. A, vol. 18, no. 6, pp. 1312-1324, June 2001.

[6] X. Zhu and P. Milanfar, "Image Reconstruction from Videos Distorted by Atmospheric Turbulence," Proc. SPIE Electronic Imaging Conf. Visual Information Processing and Comm., Jan. 2010.

[7] Shimizu, M., Yoshimura, S., Tanaka, M., and Okutomi, M., "Super-resolution from image sequence under influence of hot-air optical turbulence," CVPR 2008, 1-8 (June 2008).

[8] Fried, D. L., "Probability of getting a lucky short-exposure image through turbulence," Optical Society of America, Journal 68, $1651-1658(1978)$

[9] John, S. and Vorontsov, M. A., "Multi-frame selective information fusion from robust error estimation theory," IEEE Transactions on Image Processing 14, 577-584 (May 2005).

[10] Aubailly, M., Vorontsov, M. A., Carhat, G. W., and Valley, M. T, "Automated video enhancement from a stream of atmospherically-distorted images: the lucky-region fusion approach,” Proceedings of SPIE 7463 (2009). 